\title{
An Approach for Predicting the Shape and Size of a Buried Basic Object on Surface Ground Penetrating Radar System
}

\author{
Nana Rachmana Syambas \\ School of Electrical Engineering and Informatics, Bandung Institute of Technology, Bandung 40132, Indonesia \\ Correspondence should be addressed to Nana Rachmana Syambas, nana@telecom.ee.itb.ac.id
}

Received 2 March 2012; Revised 4 May 2012; Accepted 26 June 2012

Academic Editor: Francesco Soldovieri

Copyright ( $) 2012$ Nana Rachmana Syambas. This is an open access article distributed under the Creative Commons Attribution License, which permits unrestricted use, distribution, and reproduction in any medium, provided the original work is properly cited.

Surface ground-penetrating radar (GPR) is one of the radar technology that is widely used in many applications. It is nondestructive remote sensing method to detect underground buried objects. However, the output target is only hyperbolic representation. This research develops a system to identify a buried object on surface GPR based on decision tree method. GPR data of many basic objects (with circular, triangular, and rectangular cross-section) are classified and extracted to generate data training model as a unique template for each type of basic object. The pattern of object under test will be known by comparing its data with the training data using a decision tree method. A simple powerful algorithm to extract feature parameters of object which is based on linear extrapolation is proposed. The result showed that tested buried basic objects can be correctly predicted and the developed system works properly.

\section{Introduction}

Surface ground-penetrating radar (GPR) which has evolved as popular technology for nondestructive testing method has been widely used for the detection of underground buried objects since 1980. The interesting applications are for the detection of underground infrastructure [1] and the landmine [2]. GPR transmits short pulses of high frequency electromagnetic into the ground. These waves propagate with a velocity that depends on the dielectric property of medium. If the waves encounter a buried object with different reactive indices, some of the waves are reflected back and the receiver processes them to create a hyperbolic image as the object representation. In order to create an image, the GPR transmits electromagnetic pulses at a certain frequency for a certain time slot and samples the responses as an A-scan corresponding to a single position of the GPR. By moving the GPR in $x$ direction, a collection of these Ascans called a B-scan which represent different GPR positions are constructed. Then it is processed to become a hyperbolic image. Ideally, the imaging process of GPR data is described in Figure 1.
Automatic generation of hyperbolic image from B-scan data has been widely reported using various methods and techniques such as Hough transform [3, 4], statistical and probability $[5,6]$, genetic algorithm [7], neural networks $[4,8,9]$, and the latest technique is compressive sensing [10].

Figure 2 shows the B-scan of GPR data for basic circular and rectangular objects. From the image, it is hard to recognize the shape of the object. Image of an object generated by GPR is not corresponding to its geometrical representation.

Regeneration of a geometric model is a much more complex procedure and is not usually attempted [11]. However, because the output target is only a hyperbolic representation, interpretation of a geometric buried object is depends on operator's expertise and experience.

Recent developments have shown that significant efforts have been focused towards the extraction of meaningful physical interpretation from GPR data. Researches on generating geometrical representation have been reported in [1214]. Unfortunately, these reports are based on diffraction tomography or cross-hole GPR method, which is not practical to drill holes and is time consuming [15]. 


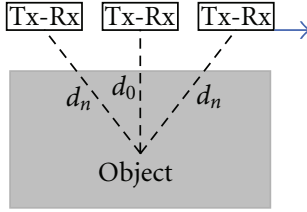

Acquisition

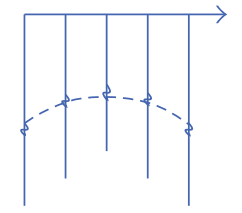

B-scan of an object

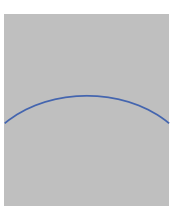

Image of an object

FIGURE 1: Imaging process of GPR data.
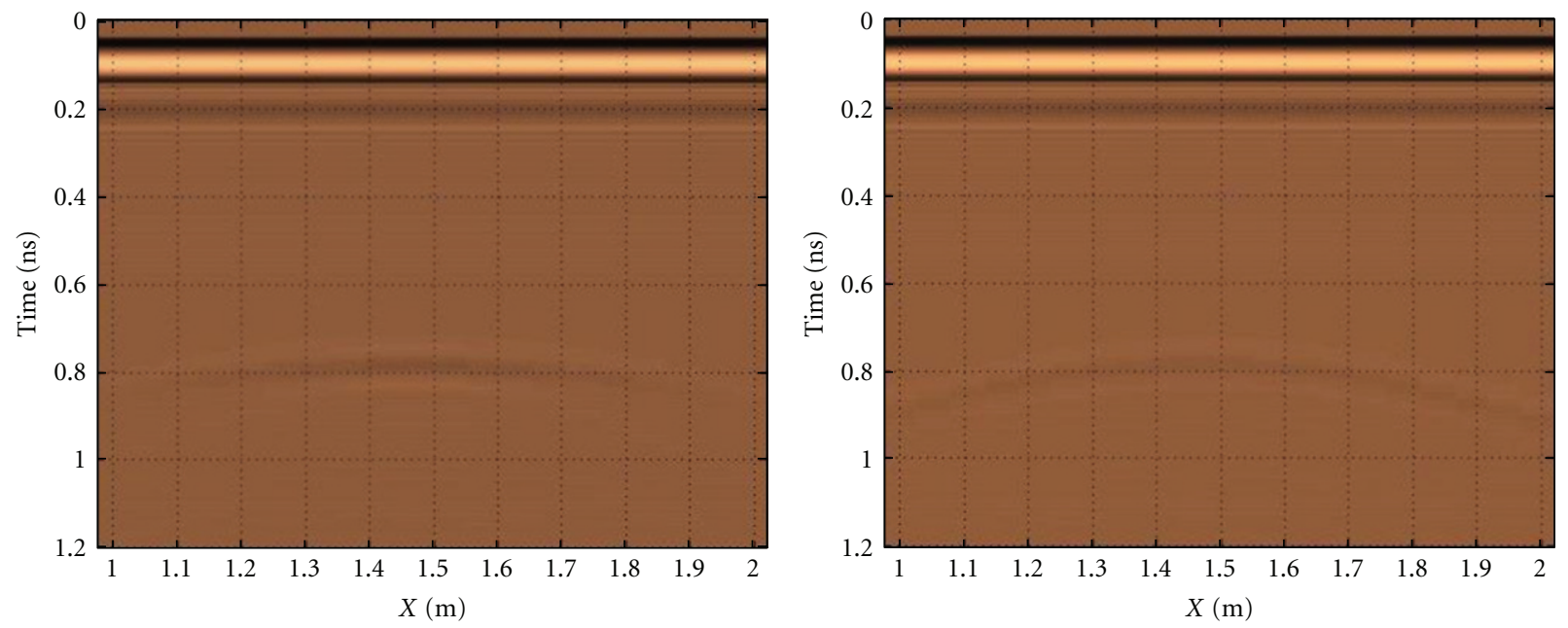

Figure 2: B-scan of GPR data for basic circular and rectangular objects.

This research tries to interpret geometrical representation from a B-scan of GPR data based on template matching. The pattern of object under test will be interpreted by comparing its data with the training data using a simple decision tree method.

\section{The Proposed System}

Proposed system for identification [16] is shown in Figure 3. Firstly an output GPR (a file*a.out) is extracted to generate reflected signal $E_{(z)}(\mathrm{V} / \mathrm{m})$ as function of time (ns). The difference of the output GPR format and the input format of the C.45 [17] software tool, needs a program to convert those formats. The program is based on Java called DataCreator, NamesCreator, TstCreator, and AhCreator which generates a file*.data, a file*.names, a file*.tst, and file*.ah, respectively. The first two files are needed to generate the training data and the last twofiles are needed to generate and to test the test data.

The C.45 software tool needsa built-tree of training data to predict a decision. Generation of built-tree can be done after file*.data and file*.names have been generated. Values of training data are calculated to determine entropy and gain ratio for each attribute. Entropy is used to measure how informative is a node in a tree, and gain ranks attribute to build a decision tree of the training data.

Interpretation of a test data can be done after the file*.tst is generated. Using the built-tree, a tested data record that has unknown attribute values can be classified by estimating the probability of the various possible results.

Finally the ResultShower.jar program converts the output decision of C.45 in text form to visual pattern form to show the visual of the tested unknown object. These steps of the process are summarized in algorithm 1.

As example on this experiment, training data consist of 30 samples of three types (circular, triangular, and rectangular) of object data. For each type object data has 10 different size samples $(r: 7,10,12,13,15,17,18,19,20$, and 23). Each sample has 10180 attributes of the file*.data, so the training data has totally 305400 attributes. Figure 4 shows the built-tree of the training data.

2.1. GPR Test Range Model. The model GPR test range is a box with 3 meter length, 3 meter wide, and 2 meter high $(3 \times 3 \times 2$ meter $)$, containing a half full of sand. A $1 \mathrm{GHz}$ transmitter and receiver of GPR, which has 10 centimetre fix distance and located 20 centimetre above the sand, moves to $x$ direction in 5 centimetre intervals until 1 meter length (20 scan positions). In each interval, the pulse signal with 12 ns width is transmitted. The sand's parameter relative permittivity is 3.0, conductivity is $10^{-2}$ Siemens/meter, and relative permeability is 1 . A simple buried object (with three types of patterns: circular, triangular, or rectangular crosssection) is made from perfect conductor material and is lied in the sand at $50 \mathrm{~cm}$ depth. Figure 5 shows the model of simulation. 


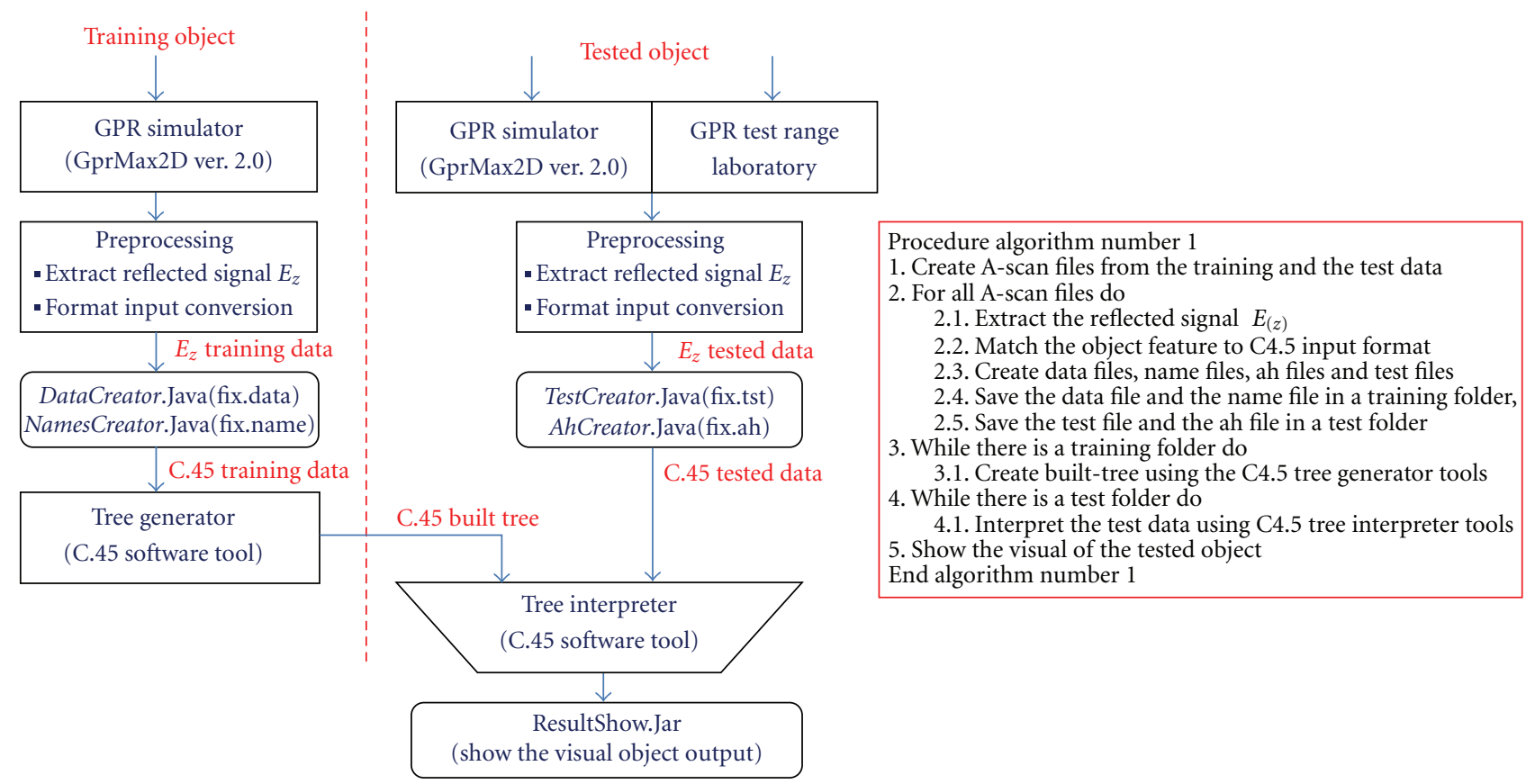

FIgURE 3: Proposed system and algorithm number 1.

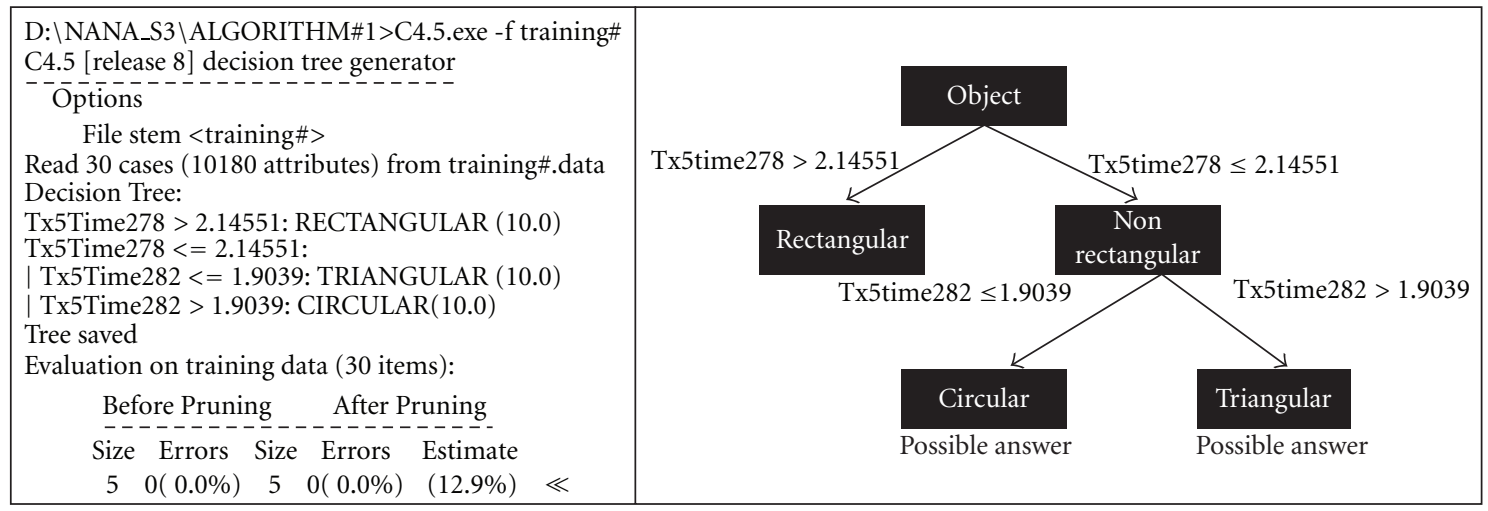

FIgURE 4: Built-tree of the training data with algorithm number 1.

TABLE 1: The test result based on algorithm number 1 .

\begin{tabular}{|c|c|c|c|c|c|c|c|c|}
\hline \multirow{2}{*}{ DUT } & \multicolumn{8}{|c|}{ Sc no. } \\
\hline & Sc no. 0 & Sc no. 1 & Sc no. 2 & Sc no. 3 & Sc no. 4 & Sc no. 5 & Sc no. 6 & Sc no. 7 \\
\hline TRI_8 & True & True & True & True & True & True & False & True \\
\hline TRI_9 & True & True & True & True & True & True & False & True \\
\hline TRI_21 & True & True & True & True & True & True & False & True \\
\hline TRI_22 & True & True & True & True & True & True & False & True \\
\hline CIR_8 & True & True & False & False & False & False & False & False \\
\hline CIR_9 & True & True & False & False & False & False & False & False \\
\hline CIR_21 & True & True & False & False & False & False & False & False \\
\hline CIR_22 & True & True & False & False & False & False & False & False \\
\hline REC_8 & True & True & False & False & True & False & False & False \\
\hline REC_9 & True & True & False & False & True & False & False & False \\
\hline REC_21 & True & True & False & False & True & False & True & False \\
\hline REC_22 & True & True & False & False & True & False & True & False \\
\hline
\end{tabular}


TABLE 2: The test result based on algorithm number 2.

\begin{tabular}{lccccccc}
\hline DUT & & & & \multicolumn{2}{c}{ Sc no. } & & \\
& Sc no. 0 & Sc no. 1 & Sc no. 2 & Sc no. 3 & Sc no. 4 & Sc no. 5 & Sc no. 6 \\
\hline TRI_8 & True & True & True & True & True & True & True \\
TRI_9 & True & True & True & True & True & True & True \\
TRI_21 & True & True & True & True & True & True & True \\
TRI_22 & True & True & True & True & True & True & True \\
CIR_8 & True & True & True & True & True & True & True \\
CIR_9 & True & True & True & True & True & True & True \\
CIR_21 & True & True & True & True & True & True & True \\
CIR_22 & True & True & True & True & True & True & True \\
REC_8 & True & True & True & True & True & True & True \\
REC_9 & True & True & True & True & True & True & True \\
REC_21 & True & True & True & True & True & True & True \\
REC_22 & True & True & True & True & True & True & True \\
\hline
\end{tabular}

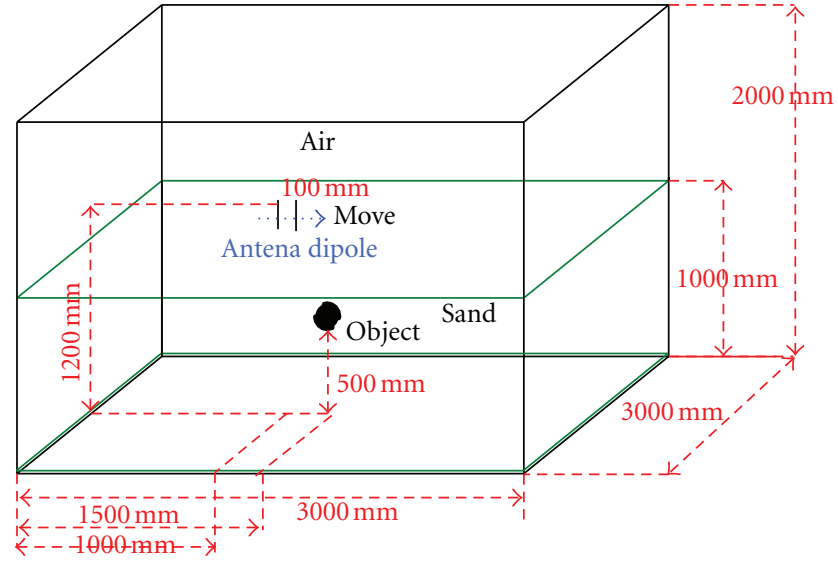

Figure 5: GPR test range model.

TABLE 3: Correction factor for all scenarios.

\begin{tabular}{lc}
\hline Scenario no. 0 & $k=1$ \\
Scenario no. 1 & $k=1.06$ \\
Scenario no. 2 & $k=0.9$ \\
Scenario no. 3 & $k=0.95$ \\
Scenario no. 4 & $k=0.8$ \\
Scenario no. 5 & $k=1.13$ \\
Scenario no. 6 & $k=0.85$ \\
Scenario no. 7 & $k=1.2$ \\
\hline
\end{tabular}

For training purpose, 30 samples of three types (circular, triangular, and rectangular) of object data are generated. Each type of object data has 10 different size samples. Test data consist of 12 samples of the three types of objects in which each type has 4 different sample sizes.

Successful GPR modelling based on the finite-difference time-domain (FDTD) theory [18] has been reported by many authors [19-22].
Giannopoulos [19] has developed GPR modelling facilities called GprMax2D/3D ver. 2.0 which are used to generate the GPR data. The output simulator can be a file*b.out and file*a.out to represent hyperbolic image in binary file and ASCII file, respectively. Sampling experiment by using real GPR tool will be done in GPR test range ICTR-ITB laboratory to validate and verify the GPR data simulator. An example of input and output GPR data simulator is shown in Figure 6. The research takes files*a.out as input of the proposed system to generate training data and tests data files.

2.2. Testing Scenarios. There are scenarios to cover both the same environment test condition and the different environment test condition. The test data for each scenario consist of 12 samples of the three types of object in which each type has 4 different sample sizes $(r: 8,9,21$, and 22). The total samples test data for all scenarios are 96 samples. These scenarios are as follows.

(1) Tested and training objects are in the same 50 centimetre depth condition.

(i) Scenario number 0. The sand and the tested object have the same parameters as in the training data (the sand: $\varepsilon_{r}=3, \mu_{r}=1$, and the object: perfect conductor). Tested and training objects have the same environmental condition.

(ii) Scenario number 1 . The sand has the same parameter as in training data and the tested object has different material (the sand: $\varepsilon_{r}=3$, $\mu_{r}=1$, and the object: fresh water, $\rho_{r}=81$ ).

(iii) Scenario number 2. The tested object has the same parameter as training data and the sand has different permittivity (sand: $\varepsilon_{r}=4, \mu_{r}=1$, and the object: perfect conductor).

(iv) Scenario number 3. The sand medium and the tested object have different parameters compared to training data (sand: $\varepsilon_{r}=4, \mu_{r}=1$, and the object: fresh water, $\left.\rho_{r}=81\right)$. 


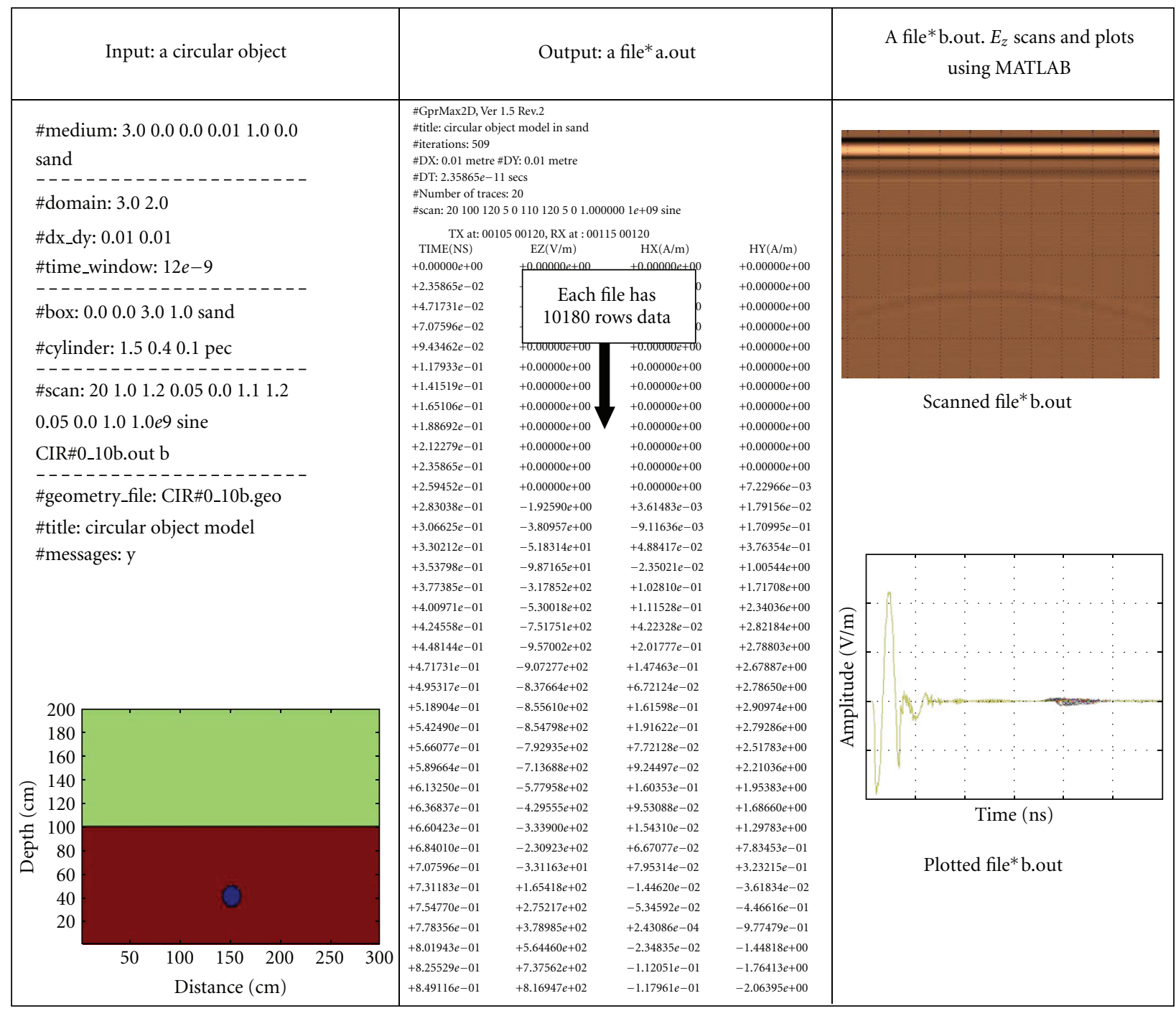

FIgUre 6: An input and an output GPR data simulator.

(2) Tested and training objects are in the different depth.

(i) Scenario number 4 . The sand and the tested object have the same parameters as in the training data (the sand: $\varepsilon_{r}=3, \mu_{r}=1$, and the object: perfect conductor), and depth of the tested object is $40 \mathrm{~cm}$.

(ii) Scenario number 5. The sand and the tested object have the same parameters as in the training data (the sand: $\varepsilon_{r}=3, \mu_{r}=1$, and the object: perfect conductor), and depth of the tested object is $55 \mathrm{~cm}$.

(iii) Scenario number 6. The sand has the same parameter as in training data and the object to be tested has different material (the sand: $\varepsilon_{r}=$ $3, \mu_{r}=1$, and the object: fresh water, $\rho_{r}=81$ ), and the depth of the tested object is $40 \mathrm{~cm}$.

(iv) Scenario number 7. The sand has the same parameter as in training data and the tested object has different material (the sand: $\varepsilon_{r}=3$, $\mu_{r}=1$, and the object: fresh water, $\rho_{r}=8$ ), and depth of the tested object is $55 \mathrm{~cm}$.

2.3. The Test Result Based on Algorithm Number 1. An example of interpretation of the test result for scenario number 6 is shown in Figure 7. This scenario in which tested and training objects are in the different environmental condition, generates the extreme result, that is, the most of interpretation is false. On the other hand, scenario number 0 , in which tested andtraining objects are in the same environmental condition, generates the best result, that, is all interpretation is true.

The complete test results are summarized in Table 1. From 96 tested data samples, the system can truly well interpret only for 50 samples (52\%). Algorithm 1, which represent direct comparison between test and training data with template matching method, has inaccurate interpretation 


\begin{tabular}{|c|c|}
\hline Device under test & Results of the interpretation \\
\hline & $\begin{array}{l}\text { D:\ALGORTM\#1 }>\text { T.exe }-\mathrm{f} \text { training\# training\#.ah } \\
\text { TRI\#1_21_40.tst } \\
\text { C4.5 [release 8] decision tree interpreter } \\
\text { - }-1--------------- \\
\text { Tx5Time278: inserting } 1.151200 \\
\text { Tx5Time282: inserting } 5.543930 \\
\text { Decision: } \\
\quad \text { CIRCULAR CF }=1.00[0.87-1.00]\end{array}$ \\
\hline & $\begin{array}{l}\text { D: } \backslash \text { ALGORTM\# } 1>\text { T.exe }-\mathrm{f} \text { training\# training\#.ah } \\
\text { CIR\#1_8_40.tst } \\
\text { C4.5 [release 8] decision tree interpreter } \\
--------------- \\
\text { Tx5Time278: inserting } 2.353900 \\
\text { Decision: } \\
\quad \text { RECTANGULAR CF }=1.00[0.87-1.00]\end{array}$ \\
\hline & $\begin{array}{l}\text { D: } \backslash \text { ALGORTM\# } 1>\text { T.exe }-\mathrm{f} \text { training\# training\#.ah } \\
\text { REC\#1_9_40.tst } \\
\text { C4.5 [release 8] decision tree interpreter } \\
- \text { Tx5Time278: inserting } 1.680600 \\
\text { Tx5Time282: inserting } 1.186690 \\
\text { Decision: } \\
\quad \text { TRIANGULAR CF }=1.00[0.87-1.00]\end{array}$ \\
\hline
\end{tabular}

Figure 7: An example of the partial test result for scenario number 6.

for condition where the tested and the training object have different environmental condition.

\section{The Feature Extraction}

An effort to enhance the performance of the system is done by adding a feature extraction of the object to algorithm 1 . This improved the algorithm called as algorithm 2 .

Investigation of reflected signal $E_{(z)}$ received by receiver for 30 samples of three types of (circular, triangular, and rectangular) object of training data carries out an important information, that is, each type of object has a unique pattern signal. In the same type of object, different size will generate different amplitude signal with the same pattern signal. This phenomenon can be seen in Figure 8 .

Afterward, the unique pattern signal of object is extracted as the objectfeature, that is, a representation of the object. To simplify the feature and to reduce the number attribute of complex extracted signal pattern, the time variable is excluded by sorting values of the $E_{(z)}$ from the smallest to the largest, and then a linear extrapolation is applied. Changing the testing condition $\left(\varepsilon_{r}, \mu_{r}, \rho_{r}\right.$, and depth) will shift the position in time axis and change the amplitude signal without changing the pattern of object.

By this process, each reflected signal pattern of an object, which in algorithm 1 has 10180 attributes, will be transformed to a straight line that only has 3 attributes, called slope of the line $(a)$, intersection point of the line with the $E_{(z)}$ axis $(b)$, and standard deviation $(s)$. Furthermore, these attributes are used as the object representations are processed
TABLE 4: Size of prediction of tested object.

\begin{tabular}{|c|c|c|c|}
\hline \multirow{3}{*}{$\begin{array}{l}\text { Size of tested } \\
\text { object } r(\mathrm{~cm})\end{array}$} & \multicolumn{2}{|c|}{ Prediction result $(\mathrm{cm})$} & \multirow{3}{*}{ Scenario } \\
\hline & Rectangular & Circular object & \\
\hline & Size $r$ & Size $r$ & \\
\hline 8 & 7.5 & 8.0 & \multirow{4}{*}{ Sc no. 0} \\
\hline 9 & 8.4 & 8.9 & \\
\hline 21 & 21.0 & 21.8 & \\
\hline 22 & 21.2 & 21.8 & \\
\hline 8 & 8.1 & 10.6 & \multirow{4}{*}{ Sc no. 4} \\
\hline 9 & 9.2 & 11.7 & \\
\hline 21 & 21.0 & 26.8 & \\
\hline 22 & 21.7 & 27.1 & \\
\hline 8 & 7.5 & 9.6 & \multirow{4}{*}{ Sc no. 5} \\
\hline 9 & 8.3 & 10.5 & \\
\hline 21 & 20.6 & 22.7 & \\
\hline 22 & 21.0 & 22.7 & \\
\hline 8 & 7.9 & 9.6 & \multirow{4}{*}{ Sc no. 2} \\
\hline 9 & 8.9 & 11.2 & \\
\hline 21 & 22.2 & 23.5 & \\
\hline 22 & 22.6 & 23.9 & \\
\hline 8 & 7.5 & 5.1 & \multirow{4}{*}{ Sc no. 1} \\
\hline 9 & 8.5 & 5.6 & \\
\hline 21 & 21.2 & 19.6 & \\
\hline 22 & 21.4 & 22.8 & \\
\hline 8 & 7.6 & 6.7 & \multirow{4}{*}{ Sc no. 7} \\
\hline 9 & 8.4 & 7.7 & \\
\hline 21 & 20.1 & 20.6 & \\
\hline 22 & 20.7 & 23.1 & \\
\hline 8 & 8.7 & 6.6 & \multirow{4}{*}{ Sc no. 6} \\
\hline 9 & 10.0 & 8.8 & \\
\hline 21 & 23.1 & 21.9 & \\
\hline 22 & 24.1 & 25.2 & \\
\hline 8 & 8.4 & 5.5 & \multirow{4}{*}{ Sc no. 3} \\
\hline 9 & 9.5 & 7.1 & \\
\hline 21 & 21.1 & 20.6 & \\
\hline 22 & 21.6 & 23.0 & \\
\hline
\end{tabular}

using template matching method by comparing attribute of tested data to training data, the same as in algorithm 1 .

Figure 9 shows algorithm 2 illustrated in each step, starting from extracting the reflected signal, extracting the feature, and matching the tested and training attribute data using decision tree to generate the interpretation result.

3.1. The Test Result Based on Algorithm Number 2. The system based on algorithm 2 is tested by the test data and scenarios the same as in the system based on algorithm number 1. An example of interpretation of the partial test result for scenario number 5 is shown in Figure 10 and all the test result is summarized in Table 2. For 96 test samples in different scenarios, the result showed that the system correctly interprets all the samples. Algorithm number 2 


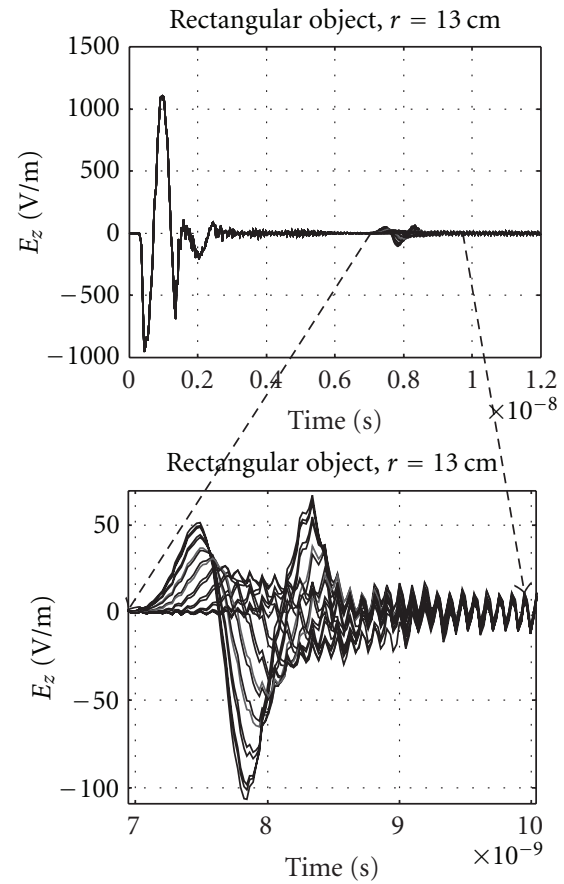

(a) Rectangular

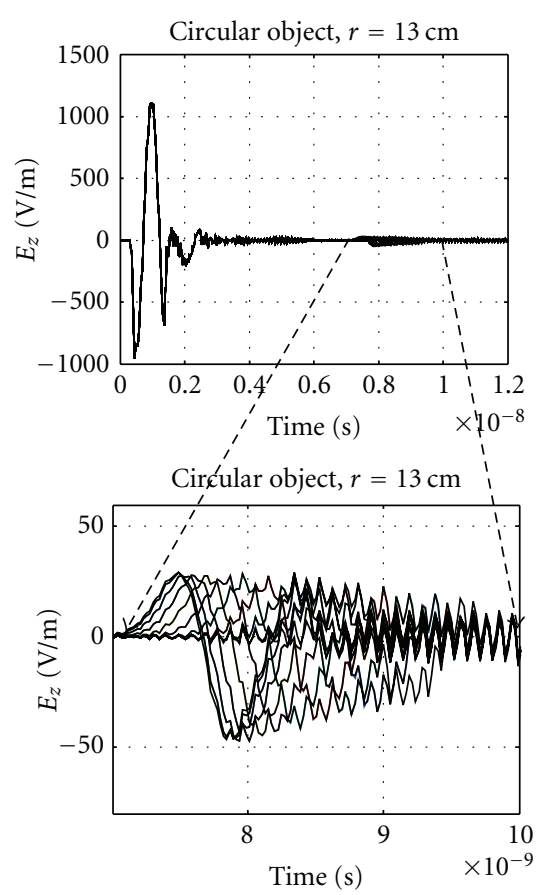

(b) Circular

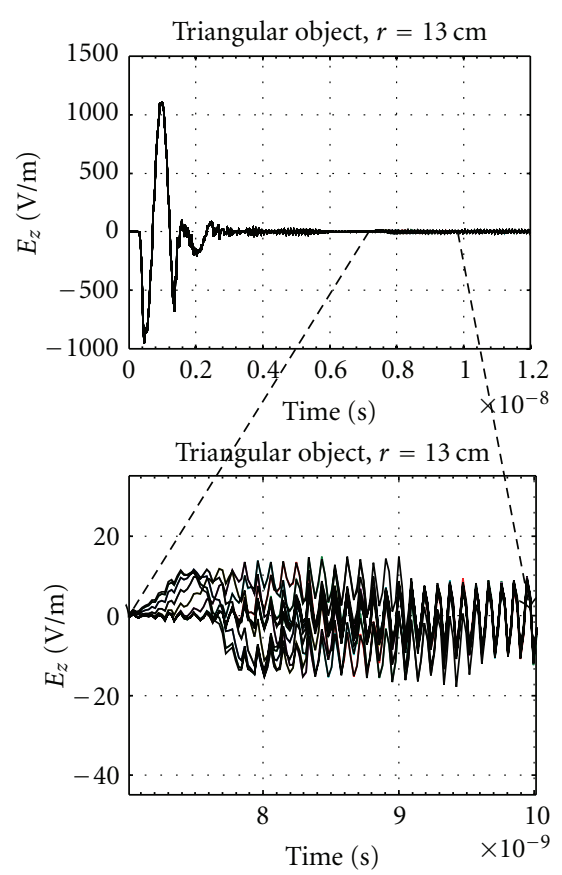

(c) Triangular

FIGURE 8: Received signal pattern for three types of the basic object.

has accurate interpretations for all environmental testing conditions.

\section{Calculation of the Object Size}

Based on reflected signal $E_{(z)}$ for 30 samples training data of three types (circular, triangular, and rectangular), the maximum and minimum signals amplitude can be determined. In the same type of object, different size will generate different amplitude signal with the same pattern signal. By linear or logarithmic extrapolation of signals amplitude, a formula to calculate another size of objects in the same object type with similar environmental condition $\left(d, \varepsilon_{r}, \mu_{r}\right.$, and $\left.\sigma_{r}\right)$ can be generated as in Figure 11. Unfortunately for the triangular object, the generated formula is constant. Constant relationship between amplitude and size of triangular shape can be due to the backscattered rays which are not directed towards the antenna.

The formula for a tested object has different condition $\left(d, \varepsilon_{r}, \mu_{r}\right.$ and $\left.\sigma_{r}\right)$ with the training object, which is need to be corrected by a correction factor as can be derived from Figure 12.

Intrinsic impedance, transmission, and reflection factors are as follows:

$$
\begin{gathered}
\eta=\sqrt{\frac{\mu}{\varepsilon}} \eta=\sqrt{\frac{\mu}{\varepsilon}}, \quad T=\frac{2 \eta_{1}}{\eta_{1}+\eta_{0}}, \quad \Gamma=\frac{\eta_{2}-\eta_{1}}{\eta_{1}+\eta_{2}}, \\
T_{u p}=\frac{2 \sqrt{1 / \varepsilon_{r 1}}}{\sqrt{1 / \varepsilon_{r 0}}+\sqrt{1 / \varepsilon_{r 1}}}=\frac{2}{1+\sqrt{\varepsilon_{r 1} / \varepsilon_{r 0}}}=\frac{2}{1+\sqrt{\varepsilon_{r 1}}},
\end{gathered}
$$

$$
\begin{aligned}
& T_{p u}=\frac{2 \sqrt{1 / \varepsilon_{r 0}}}{\sqrt{1 / \varepsilon_{r 0}}+\sqrt{1 / \varepsilon_{r 1}}}=\frac{2 \sqrt{\varepsilon_{r 1} / \varepsilon_{r 0}}}{1+\sqrt{\varepsilon_{r 1} / \varepsilon_{r 0}}}=\frac{2 \sqrt{\varepsilon_{r 1}}}{1+\sqrt{\varepsilon_{r 1}}}, \\
& T_{p b}=\frac{2 \sqrt{1 / \varepsilon_{r b}}}{\sqrt{1 / \varepsilon_{r b}}+\sqrt{1 / \varepsilon_{r 1}}}=\frac{2 \sqrt{\varepsilon_{r 1} / \varepsilon_{r b}}}{1+\sqrt{\varepsilon_{r 1} / \varepsilon_{r b}}}=\frac{2}{1+\sqrt{\varepsilon_{r b} / \varepsilon_{r p}}}, \\
& T_{b p}=\frac{2 \sqrt{1 / \varepsilon_{r 1}}}{\sqrt{1 / \varepsilon_{r 1}}+\sqrt{1 / \varepsilon_{r b}}}=\frac{2 \sqrt{\varepsilon_{r b} / \varepsilon_{r 1}}}{1+\sqrt{\varepsilon_{r b} / \varepsilon_{r 1}}}=\frac{2}{1+\sqrt{\varepsilon_{r p} / \varepsilon_{r b}}}, \\
& \Gamma_{p b}=\frac{\eta_{b}-\eta_{p}}{\eta_{b}+\eta_{p}}=\frac{\sqrt{1 / \varepsilon_{r b}}-\sqrt{1 / \varepsilon_{r p}}}{\sqrt{1 / \varepsilon_{r b}}+\sqrt{1 / \varepsilon_{r p}}}=\frac{\sqrt{\varepsilon_{r b}}-\sqrt{\varepsilon_{r p}}}{\sqrt{\varepsilon_{r b}}+\sqrt{\varepsilon_{r p}}}, \\
& \Gamma_{b p}=\frac{\eta_{p}-\eta_{b}}{\eta_{p}+\eta_{b}}=\frac{\sqrt{1 / \varepsilon_{r p}}-\sqrt{1 / \varepsilon_{r b}}}{\sqrt{1 / \varepsilon_{r p}}+\sqrt{1 / \varepsilon_{r b}}}=\frac{\sqrt{\varepsilon_{r p}}-\sqrt{\varepsilon_{r b}}}{\sqrt{\varepsilon_{r p}}+\sqrt{\varepsilon_{r b}}}, \\
& E_{z}=E_{z 0} \times e^{-2 \alpha_{p} d} \times T_{u p} \times \Gamma_{p b} \times T_{p u}+E_{z 0} e^{-2 \alpha_{p} d} e^{-2 \alpha_{b} d_{b}} \\
& \times T_{u p} \times T_{p b} \times \Gamma_{b p} \times T_{b p} \times T_{p u}, \\
& \alpha_{p}=\frac{\omega \sqrt{\mu_{r p} \varepsilon_{r p}}}{\sqrt{2}}\left[\sqrt{1+\left(\frac{\sigma_{r p}}{\omega \varepsilon_{r p}}\right)^{2}}-1\right]^{1 / 2} \frac{N p}{m}, \\
& \alpha_{b}=\frac{\omega \sqrt{\mu_{r b} \varepsilon_{r b}}}{\sqrt{2}}\left[\sqrt{1+\left(\frac{\sigma_{r b}}{\omega \varepsilon_{r b}}\right)^{2}}-1\right]^{1 / 2} \frac{N p}{m} .
\end{aligned}
$$




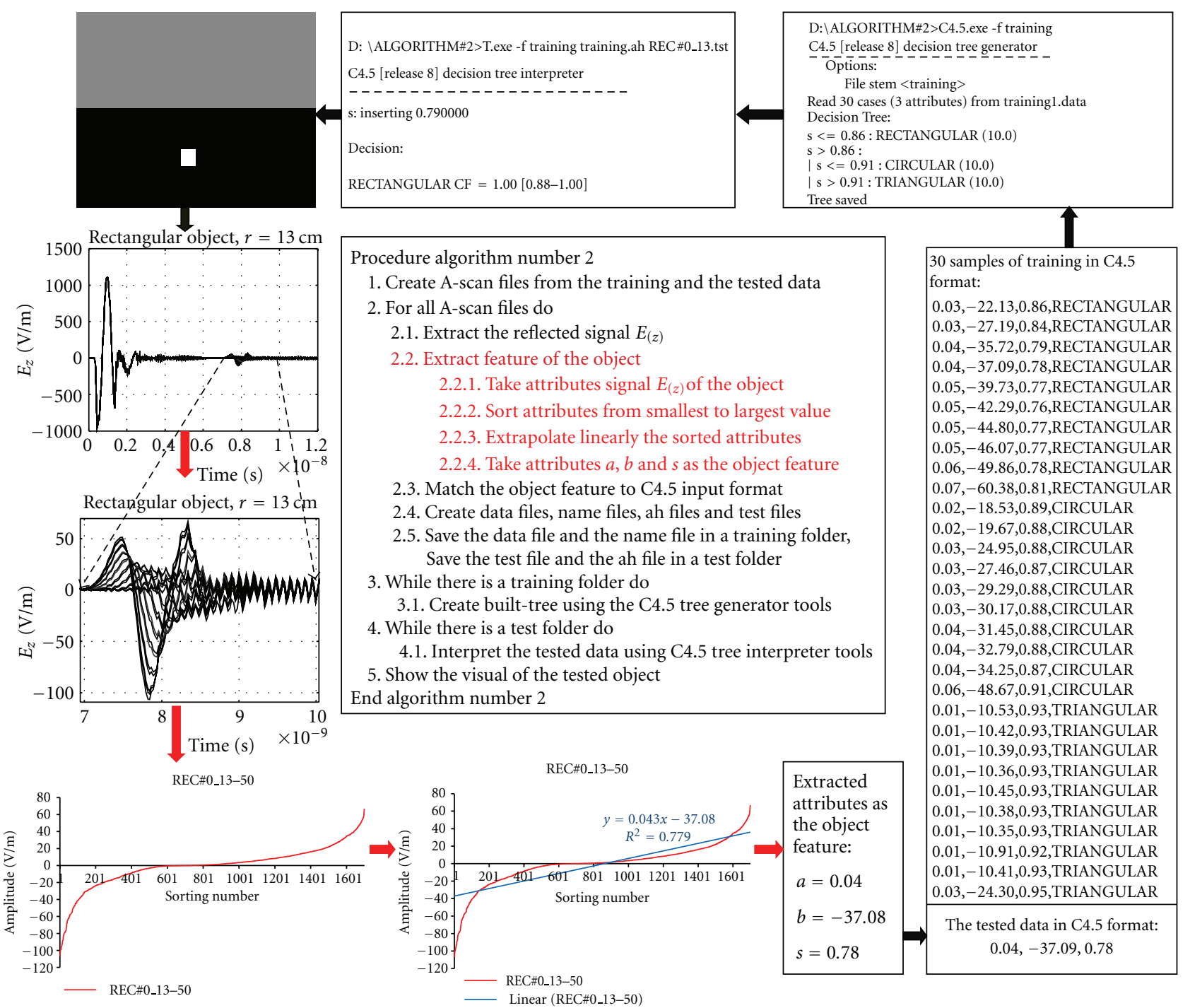

FIgURE 9: Proposed algorithm number 2 and its illustration.

The correction factor for different environmental condition

is

$$
k=\frac{\left|E_{z 1}\right|}{\left|E_{z 2}\right|}=\frac{\left(E_{z 0} \times e^{-2 \alpha_{p} d} \times T_{u p} \times \Gamma_{p b} \times T_{p u}+E_{z 0} e^{-2 \alpha_{p} d} e^{-2 \alpha_{b} d_{b}} \times T_{u p} \times T_{p b} \times \Gamma_{b p} \times T_{b p} \times T_{p u}\right) 1}{\left(E_{z 0} \times e^{-2 \alpha_{p} d} \times T_{u p} \times \Gamma_{p b} \times T_{p u}+E_{z 0} e^{-2 \alpha_{p} d} e^{-2 \alpha_{b} d_{b}} \times T_{u p} \times T_{p b} \times \Gamma_{b p} \times T_{b p} \times T_{p u}\right) 2} .
$$

As example for scenario number 1 , correction factor $k$ became

$$
\frac{\left|E_{z 1}\right|}{\left|E_{z 2}\right|}=\frac{1}{\left(\Gamma_{p b}+e^{-2 \alpha_{b} d_{b}} \times T_{p b} \times \Gamma_{b p} \times T_{b p}\right)} .
$$

For $\varepsilon_{r p}=3, d=50 \mathrm{~cm}$ and $\varepsilon_{r b}=81$, the factor correction is

$$
T_{p b}=\frac{2 \sqrt{1 / \varepsilon_{r b}}}{\sqrt{1 / \varepsilon_{r b}}+\sqrt{1 / \varepsilon_{r 1}}}=\frac{2 \sqrt{\varepsilon_{r 1} / \varepsilon_{r b}}}{1+\sqrt{\varepsilon_{r 1} / \varepsilon_{r b}}}
$$

$$
\begin{gathered}
=\frac{2}{1+\sqrt{\varepsilon_{r b} / \varepsilon_{r p}}}=\frac{2}{1+\sqrt{81 / 3}}=0.323, \\
T_{b p}=\frac{2 \sqrt{1 / \varepsilon_{r 1}}}{\sqrt{1 / \varepsilon_{r 1}}+\sqrt{1 / \varepsilon_{r b}}}=\frac{2 \sqrt{\varepsilon_{r b} / \varepsilon_{r 1}}}{1+\sqrt{\varepsilon_{r b} / \varepsilon_{r 1}}} \\
=\frac{2}{1+\sqrt{\varepsilon_{r p} / \varepsilon_{r b}}}=\frac{2}{1+\sqrt{3 / 81}}=1.5
\end{gathered}
$$




\begin{tabular}{|c|c|}
\hline Device under test & Results of the interpretation \\
\hline & $\begin{array}{l}\text { D:\ALGORTM\#2>T.exe }-\mathrm{f} \text { training training.ah } \\
\text { TRI\#0_21_55.tst } \\
\text { C4.5 [release 8] decision tree interpreter } \\
\overline{\text { s: inserting } 0.930000} \\
\text { Decision: } \\
\quad \text { TRIANGULAR CF }=1.00[0.88-1.00]\end{array}$ \\
\hline & \begin{tabular}{l} 
D:\ALGORTM \#2>T.exe $-\mathrm{f}$ training training.ah \\
CIR\#0_8_55.tst \\
C4.5 [release 8] decision tree interpreter \\
\hdashline---------1.00 \\
s: inserting 0.890000 \\
Decision: \\
$\quad$ CIRCULAR CF $=1.00[0.88-1.00]$
\end{tabular} \\
\hline & $\begin{array}{l}\text { D:\ALGORTM\#2 }>\text { T.exe }-\mathrm{f} \text { training training.ah } \\
\text { REC\#0_9_55.tst } \\
\text { C4.5 [release 8] decision tree interpreter } \\
-2 \text { inserting } 0.830000 \\
\text { Decision: } \\
\quad \text { RECTANGULAR CF }=1.00[0.88-1.00]\end{array}$ \\
\hline
\end{tabular}

Figure 10: An example of the partial test result for scenario number 5.

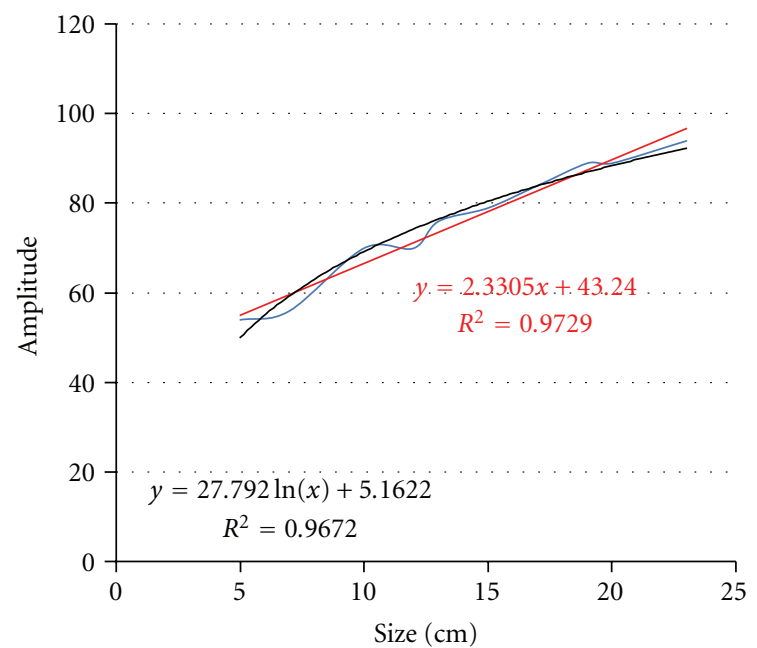

Circular object

$$
\begin{aligned}
& y=2.33 x+43.24 \\
& y=27.79 \ln (x)+5.16
\end{aligned}
$$

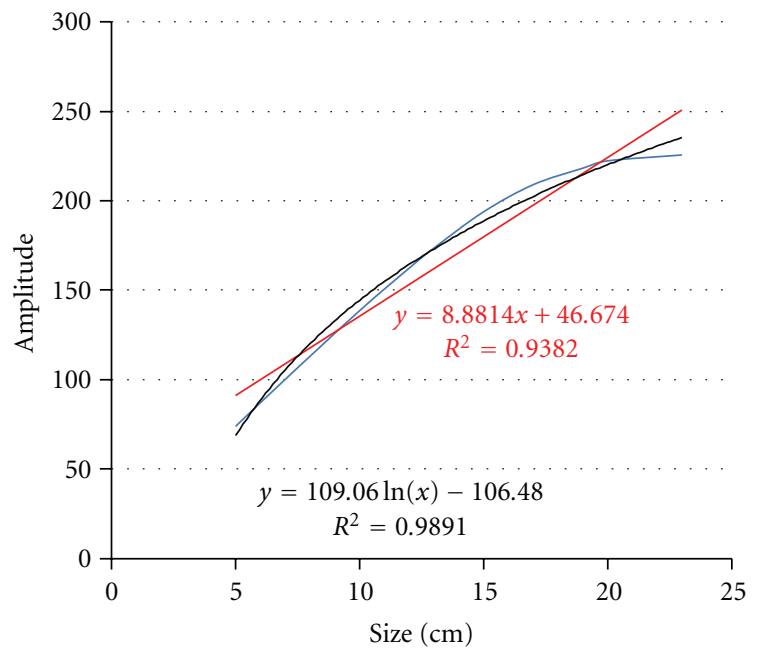

Rectangular object

Triangular object

$y \approx$ constant $=32$

FIGURE 11: Relationship of amplitude and size for same type of the object.

$$
\begin{gathered}
\Gamma_{p b}=\frac{\sqrt{\varepsilon_{r b}}-\sqrt{\varepsilon_{r p}}}{\sqrt{\varepsilon_{r b}}+\sqrt{\varepsilon_{r p}}}=\frac{\sqrt{81}-\sqrt{3}}{\sqrt{81}+\sqrt{3}}=0.677, \\
\Gamma_{p b}=\frac{\sqrt{\varepsilon_{r p}}-\sqrt{\varepsilon_{r b}}}{\sqrt{\varepsilon_{r p}}+\sqrt{\varepsilon_{r b}}}=\frac{\sqrt{3}-\sqrt{81}}{\sqrt{81}+\sqrt{3}}=-0.677, \\
\alpha_{b}=\frac{\omega \sqrt{\mu \varepsilon}}{\sqrt{2}}\left[\sqrt{1+\left(\frac{\sigma}{\omega \varepsilon}\right)^{2}}-1\right]^{1 / 2}
\end{gathered}
$$$$
=\frac{2 \pi \times 10^{9} \sqrt{81}}{\sqrt{2} \times 3 \times 10^{8}}\left[\sqrt{1+(0.0022)^{2}}-1\right]^{1 / 2}=0.2,
$$$$
\frac{\left|E_{z 1}\right|}{\left|E_{z 2}\right|}=k=\frac{1}{\left(0.677+e^{-2(0.2) 0.5} \times 0.323 \times 0.677 \times 1.5\right)}
$$$$
=1.06 \text {. }
$$ 


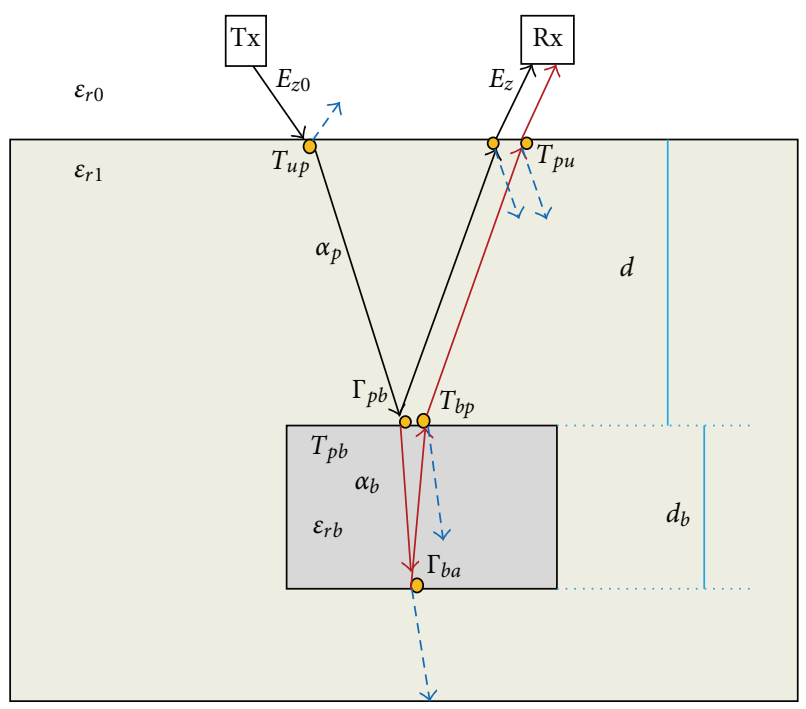

FIgURE 12: Reflected signal of nonperfect conductor object.
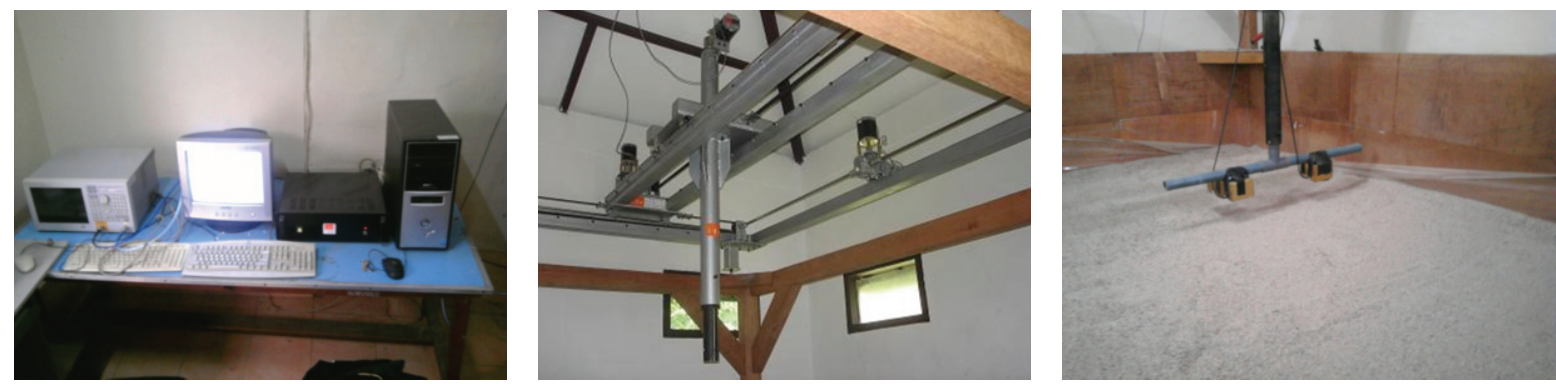

FIGURE 13: GPR test range in ICTR-ITB laboratory.

Table 3 shows that the correction factor for all scenarios has been investigated in this research.

Using the formula and the correction factor, size of tested object can be predicted. Table 4 shows the prediction result for rectangular and circular tested object. Unfortunately, due to the constant relationship between signal amplitude and object size in generated formula for triangular object, this approach did not success in predicting the triangular tested object. The overestimate or underestimate size prediction for the circular and rectangular objects occur due to the linear or logarithmic approximation of the experimental result graph as shown in Figure 11.

\section{Testing Measurement in the Laboratory}

Testing data based on sampling experiment using real GPR tool has been done in GPR test range at ICTR-ITB laboratory. The tool consists of test range, GPR scanner, spectrum analyser, and personal computer with the software tools for controlling scanner and processing data. Figure 13 shows the GPR test range in ICTR-ITB laboratory.

Three types objects are tested. These objects are buried objects (circular, triangular, and rectangular cross-section with diameter 9,20 , and $8 \mathrm{~cm}$, resp.). The object made from perfect conductor material is buried in the sand at 35, 25, and $25 \mathrm{~cm}$ depth, respectively. Figure 14 shows the measurement result for the circular object.

From Figure 15 which represents the zooming and adding cursor on B-scan, the depth of buried object as the top of hyperbolic line in $y$ axis is shown by the cursor on 517 , surface of the sand on 491, and position of the floor on 566. The real height of the sand is $100 \mathrm{~cm}$ represented by value $566-491=75$ point and the position of the object is represented by value $566-517=26$ point. So, the real position of the buried object is $26 / 75 \times 100 \mathrm{~cm}=34.7 \mathrm{~cm}$ depth.

Algorithm number 2 has been applied to the measured tested data. The reflected signal received by GPR receiver that is represented by $20 \mathrm{~A}$-scan of 20 point trace experiment result is extracted to get only the reflected signal of buried object by capturing the reflected signal in the axis range where the hyperbolic line is located and then converting the signal to the binary data in ASCII file for further manipulation.

By sorting values of the binary data from the smallest to the largest and then applying a linear extrapolation, the feature parameter of the buried object can be obtained, that is, $a=0.00007, b=0.0307$, and $c=0.8829$. 


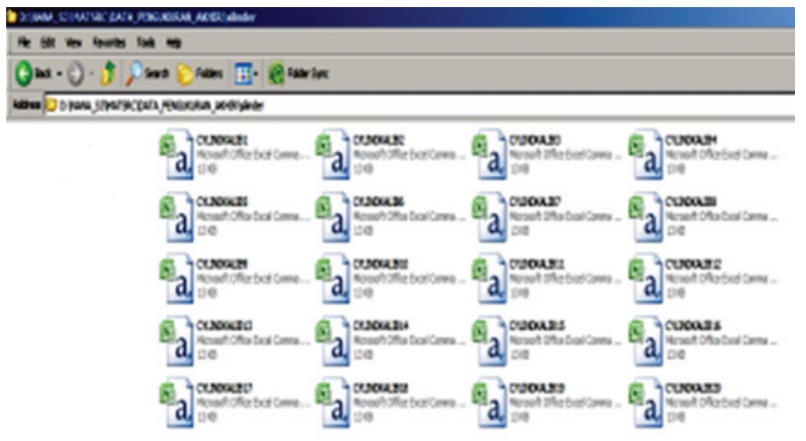

(a) Files A-scan of 20 trace points

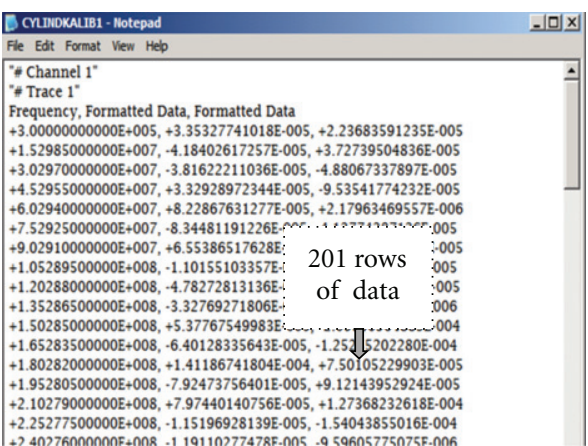

(b) A-scan for the first trace

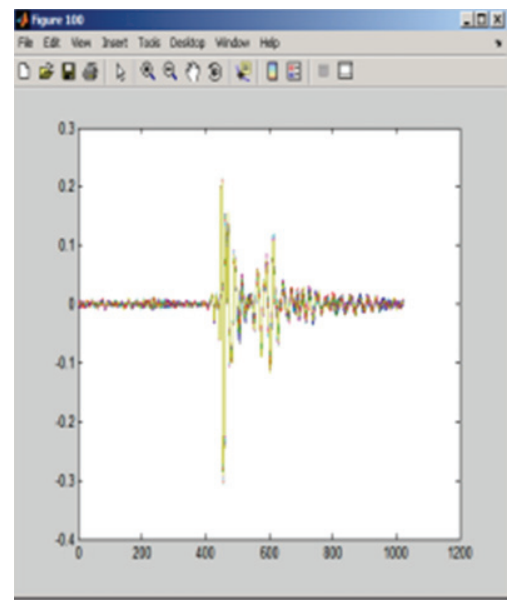

(c) The received signal $\left(E_{z}\right)$

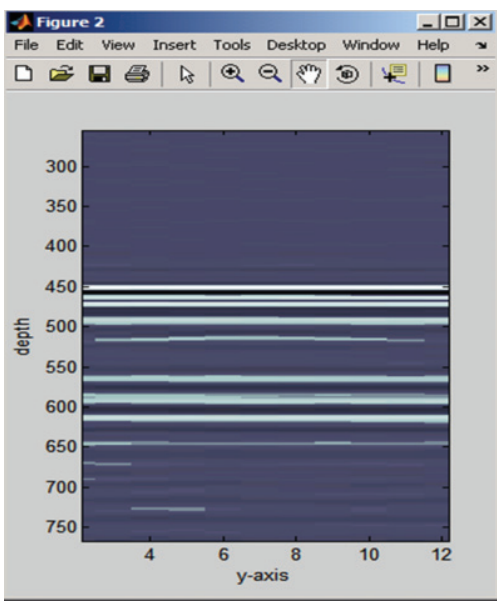

(d) The raw B-scan

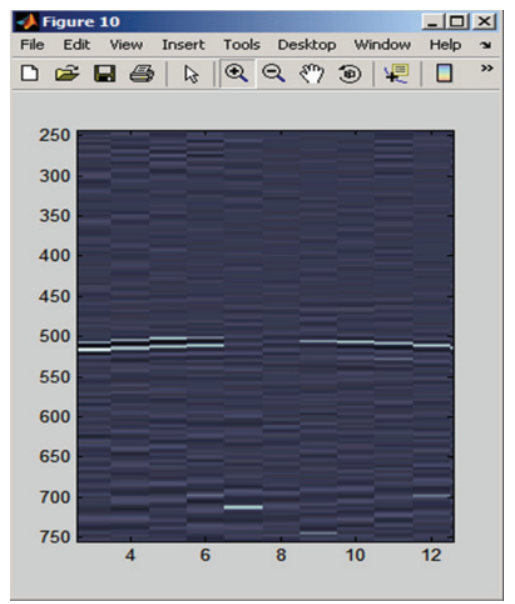

(e) The processed B-scan

Figure 14: A-scan and B-scan of the tested circular object.

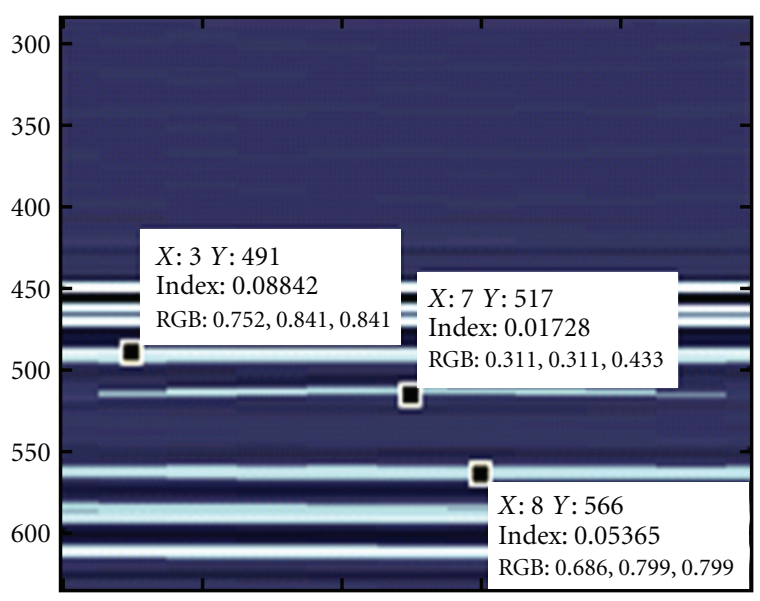

FIGURE 15: Zooming and marking the B-scan.

Matching the feature parameter with the built-tree that has been generated from training data using the C.45 tool as illustrated in Figure 9 will generate a decision of buried object interpretation. The extract feature parameter and the interpretation result of this testing are shown in Figure 16.

The same process is applied to the measured tested data for triangular and rectangular objects, generating feature parameter with values of $a=0.0001, b=0.048$, and $c=$ 0.9524 , and values of $a=0.0001, b=0.055$, and $c=0.8592$, respectively.

The amplitude of extracted signal $E_{z}$ for circular object is $119 \mathrm{mV} / \mathrm{m}$. Entering this value to the equation $y=$ $2.33 x+43.24$ results in the size value, $x$ of $32.5 \mathrm{~cm}$. The testing experiment condition in laboratory is different from training condition, so the size value should be corrected using correction factor derives from (2) as follows:

$$
\begin{gathered}
k=\frac{E_{z 01} \times e^{-2 \alpha_{p 1} d 1} \times T_{u p 1} \times T_{p u 1}}{E_{z 02} \times e^{-2 \alpha_{p 2} d 2} \times T_{u p 2} \times T_{p u 2}}, \\
d_{1}=50, \quad d_{2}=35, \quad \varepsilon_{0}=8.86 \times 10^{-12}, \\
\varepsilon_{r 1}=3, \quad \sigma=0.01, \quad \varepsilon_{r 2}=5, \quad \sigma=0.01, \\
k=\frac{1 \times e^{-2(1.09)(0.5)} \times 0.732 \times 1.268}{\sqrt{5} \times e^{-2(0.84)(0.35)} \times 0.618 \times 1.382}=0.29 .
\end{gathered}
$$

The size prediction of the test circular object can be derived by multiplying $x$ by factor $k$, that is, $0.29 \times 32.5=9.4 \mathrm{~cm}$.

Similar process can be applied to rectangular and triangular tested object. All the test result of measured test data in the ICTR-ITB laboratory can be summarized in Figure 17. 

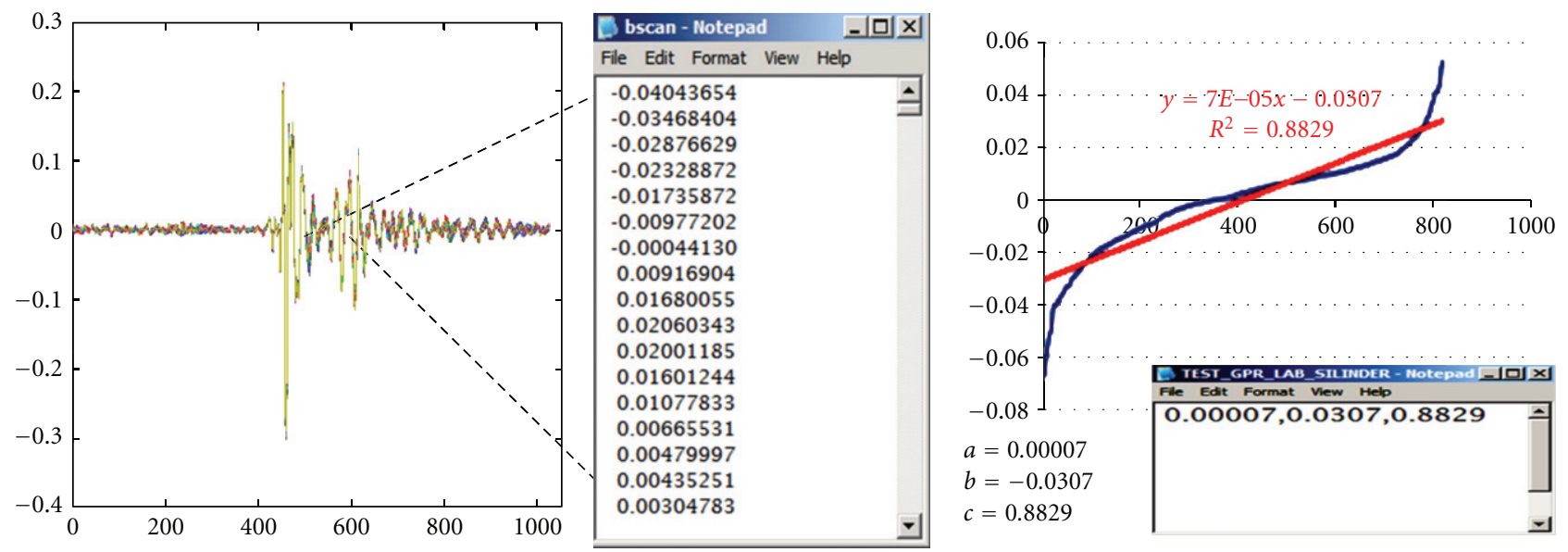

Gi) C:WTINOWS|system32/cmd.exe

D: \NANA_S3\C45\ALGORTM\#2>T.exe -f training training.ah TEST_GPR_LAB_SILINDER.tst

C4.5 [release 8] decision tree interpreter

Sun 0ct 17 07:07:32 2010

s: inserting 0.882900
Decision:
CIRCULAR CF $=1.00$ [ $0.87-1.00]$

FIGURE 16: Feature extraction and test result of the circular object.

\begin{tabular}{|c|c|c|c|c|c|}
\hline \multicolumn{3}{|c|}{ Object under test } & \multicolumn{3}{|l|}{ Test result } \\
\hline Buried object & $\begin{array}{l}\text { Depth } \\
(\mathrm{cm})\end{array}$ & $\begin{array}{l}\text { Size } \\
(\mathrm{cm})\end{array}$ & Results of the interpretation & $\begin{array}{l}\text { Depth } \\
(\mathrm{cm})\end{array}$ & $\begin{array}{c}\text { Size } \\
(\mathrm{cm})\end{array}$ \\
\hline & 35 & 9 & $\begin{array}{l}\text { D: } \mid \text { C45 } \backslash \text { ALGORTM\#2>T.exe }-\mathrm{f} \text { training } \\
\text { training.ah TEST SILINDER.tst } \\
\text { C4.5 [release } 8 \text { ] decision tree interpreter } \\
\text { s: inserting } 0.882900 \\
\text { Decision: } \\
\quad \text { CIRCULAR CF }=1.00[0.87-1.00]\end{array}$ & 34.7 & 9.4 \\
\hline & 25 & 20 & $\begin{array}{l}\text { D:IC45\ALGORTM\#2>T.exe }-\mathrm{f} \text { training } \\
\text { training.ah TEST _LIMAS.tst } \\
\text { C4.5 [release 8] decision tree interpreter } \\
- \text { s: inserting } 0.952400 \\
\text { Decision: } \\
\quad \text { TRIANGULAR CF }=1.00[0.87-1.00]\end{array}$ & 25.6 & - \\
\hline & 25 & 8 & 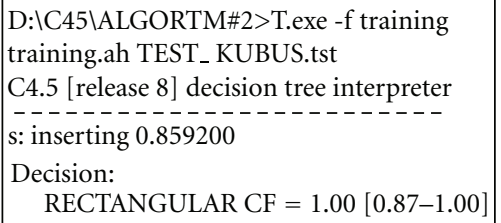 & 25.3 & 7.7 \\
\hline
\end{tabular}

FIGURE 17: The test result of measured data in the ICTR_ITB Laboratory. 


\section{Conclusions}

This paper proposed a system for identifying and predicting target shape and size of buried basic object on surface ground-penetrating radar system. Feature parameters of object are extracted by using simple proposed algorithm which is based on linear extrapolation. 96 samples of basic objects which are laid underground and have both same and different condition with the data training are tested. Results show that all basic objects can be correctly interpreted and the proposed algorithm number 2 can improve the performance of the shape object interpretation from $52 \%$ to $100 \%$. Three sampling experiments of buried object have been done in GPR test range ICTR-ITB laboratory and the result of the test showed that the developed system works properly.

The proposed method is appropriate to be implemented in the industry to enhance the capability of GPR tools. The next output GPR target is not only in a hyperbolic representation but also can directly interpret the shape and the size of the detected object. The main application is for the underground utility infrastructure detection and interpretation such as the telecommunication and electrical cable and duct which have regular shape and size of the rectangular or circular cross-section object.

However, there is a weakness in predicting the size of triangular object due to the constant relationship between amplitude and size of the triangular shape that is generated from B-scan GPR data. For further research, instead of Bscan GPR data, using C-scan GPR data as the system input might solve this problem, even though the generation of $\mathrm{C}$ scan GPR data needs more time.

\section{References}

[1] P. D. Beukelaar, J. Dijkstra, M. Meinster, and P. Wilms, "Locating a buried culvert and the detection of underground cables and pipelines using GPR," in Proceedings of the 10th European Meeting of Environmental and Engineering Geophysics, Utrecht, The Netherlands, September 2004.

[2] L. Capineri, S. Ivashov, T. Bechtel et al., "Comparison of GPR sensor types for landmine detection and classification," in Proceedings of the 12th International Conference on Ground Penetrating Radar, Birmingham, UK, June 2008.

[3] N. Aggarwal and W. C. Karl, "Line detection in images through regularized hough transform," Journal of IEEE Transactions on Image Processing, vol. 15, no. 3, pp. 582-591, 2006.

[4] S. Shihab and W. Al-Nuaimy, "Image processing and neural network techniques for automatic detection and interpretation of GPR," http://www.wseas.us/e-library/conferences/ crete2002/papers/444-717.pdf.

[5] M. Nishimoto and K. Shimo, "A Method for detecting shallowly buried landmiles using GPR signatures," in Proceedings of the International Symposium on Antennas and Propagation (ISAP '04), Sendai, JAPAN, 2004.

[6] X. Xu, E. L. Miller, C. M. Rappaport, and G. D. Sower, "Statistical method to detect subsurface objects using array ground-penetrating radar data," Journal of IEEE Transactions on Geoscience and Remote Sensing, vol. 40, no. 4, pp. 963-976, 2002.

[7] E. Pasolli, F. Melgani, M. Donelli, R. Attoui, and M. de Vos, "Automatic detection and classification of buried objects in
GPR images using genetic algorithms and support vector machines," in Proceedings of the IEEE International Geoscience and Remote Sensing Symposium (IGARSS '08), pp. II525II528, Boston, Mass, USA, July 2008.

[8] P. Gamba and S. Lossani, "Neural detection of pipe signatures in ground penetrating radar images," IEEE Transactions on Geoscience and Remote Sensing, vol. 38, no. 2, pp. 790-797, 2000, http://www.ieeexplore.ieee.org/.

[9] H. S. Youn and C. C. Chen, "Automatic GPR target detectiion and clutter reduction using neural network," in 9th International Conference on Ground Penetrating Radar, Proceedings of SPIE, pp. 579-582, Santa Barbara, Calif, USA, April 2002.

[10] A. C. Gurbuz, Feature detection algorithms in computer images [Ph.D. thesis], Georgia Institute of Technology, Atlanta, Ga, USA, 2008.

[11] D. J. Daniels, Ed., Ground Penetrating Radar, The Institution of Electrical Engineering, 2nd edition, 2004.

[12] A. J. Devaney, "Geophysical diffraction tomography," IEEE Transactions on Geoscience and Remote Sensing, vol. 22, no. 1, pp. 3-13, 1984.

[13] E. M. Johansson and J. E. Mast, “Three-dimensional groundpenetrating radar imaging using multifrequency diffraction tomography," in Advanced Microwave and Millimeter-Wave Detectors, Proceedings of SPIE, pp. 196-203, July 1994.

[14] D. Laksameethanasan, 3D Modelling and Imaging Based on Transmission Diffraction Tomography and Algebraic Reconstruction Techniques as Applied in NDT, University of Kassel, Germany, 2004.

[15] N. Rachmana, N. Iftari, Sugihartono, and A. B. Suksmono, "Comparison of surface and cross-hole tomography radar," in Proceedings of the International Conference on Electrical Engineering and Informatics, vol. 1, no. 172-175, June 2007.

[16] N. Rachmana, Sugihartono, Hendrawan, and A. B. Suksmono, "Interpretation target pattern of a buried basic object on surface GPR system," International Journal on Electrical Engineering and Informatics, vol. 1, no. 1, pp. 53-63, 2009.

[17] J. R. Quinlan, C4. 5: Program For Machine Learning, Morgan Kauffman, 1993.

[18] K. S. Yee, "Numerical solution of initial boundary value problems involving Maxwell's equation in isotropic media," Journal of IEEE Transactions on Antennas Propagation, vol. 14, no. 3, pp. 302-307, 1996.

[19] A. Giannopoulos, “GprMax2D/3D, User's Guide," 2005, http://www.gprmax.org/.

[20] A. Giannopoulos, "Modelling ground penetrating radar by GprMax," Construction and Building Materials, vol. 19, no. 10, pp. 755-762, 2005.

[21] J. Irving and R. Knight, "Numerical modeling of groundpenetrating radar in 2-D using MATLAB," Computers \& Geosciences, vol. 32, no. 9, pp. 1247-1258, 2006.

[22] J. D. Irving and R. J. Knight, "Numerical simulation of antenna transmission and reception for crosshole ground-penetrating radar," Journal of Geophysics, vol. 71, no. 2, pp. K37-K45, 2006. 

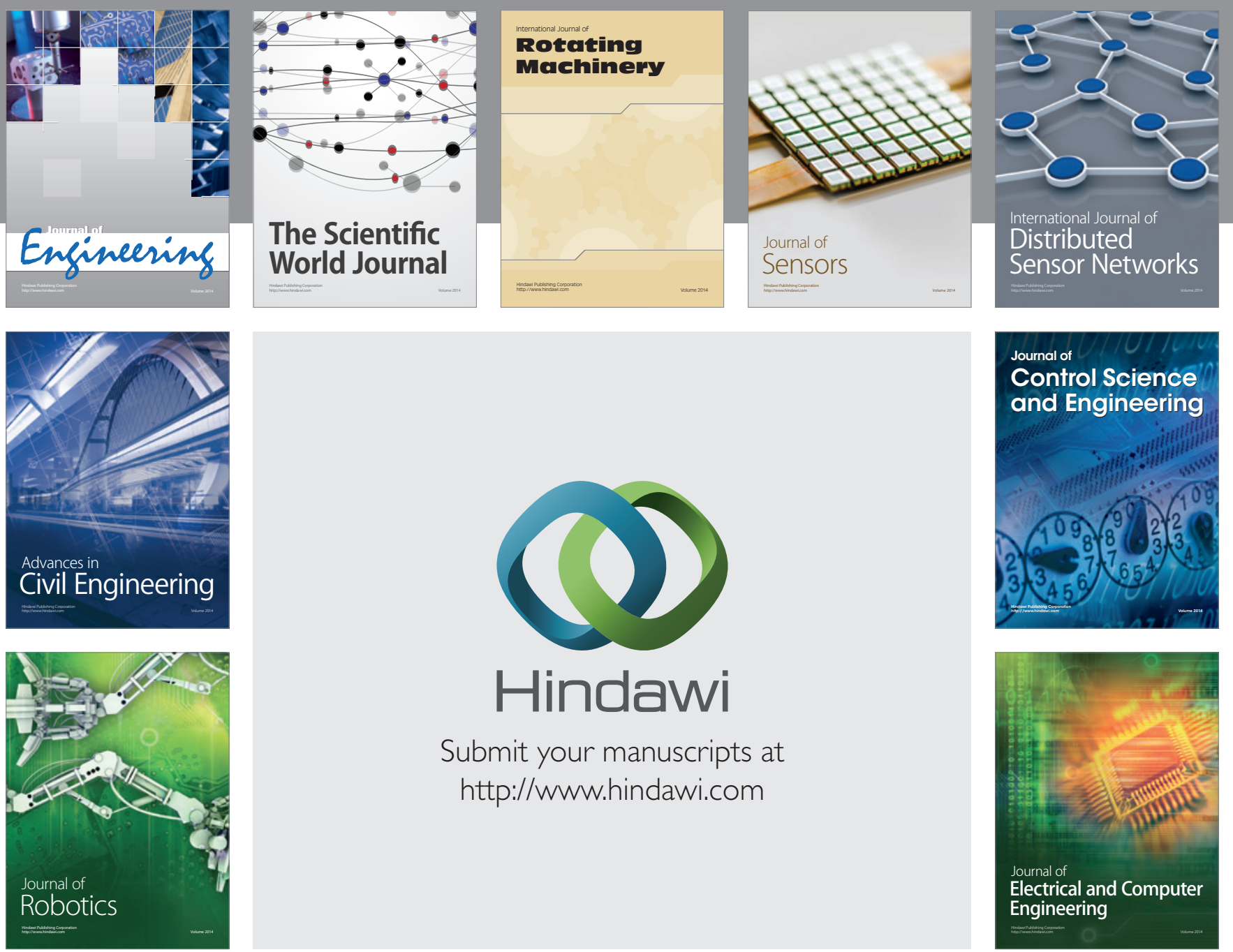

Submit your manuscripts at

http://www.hindawi.com
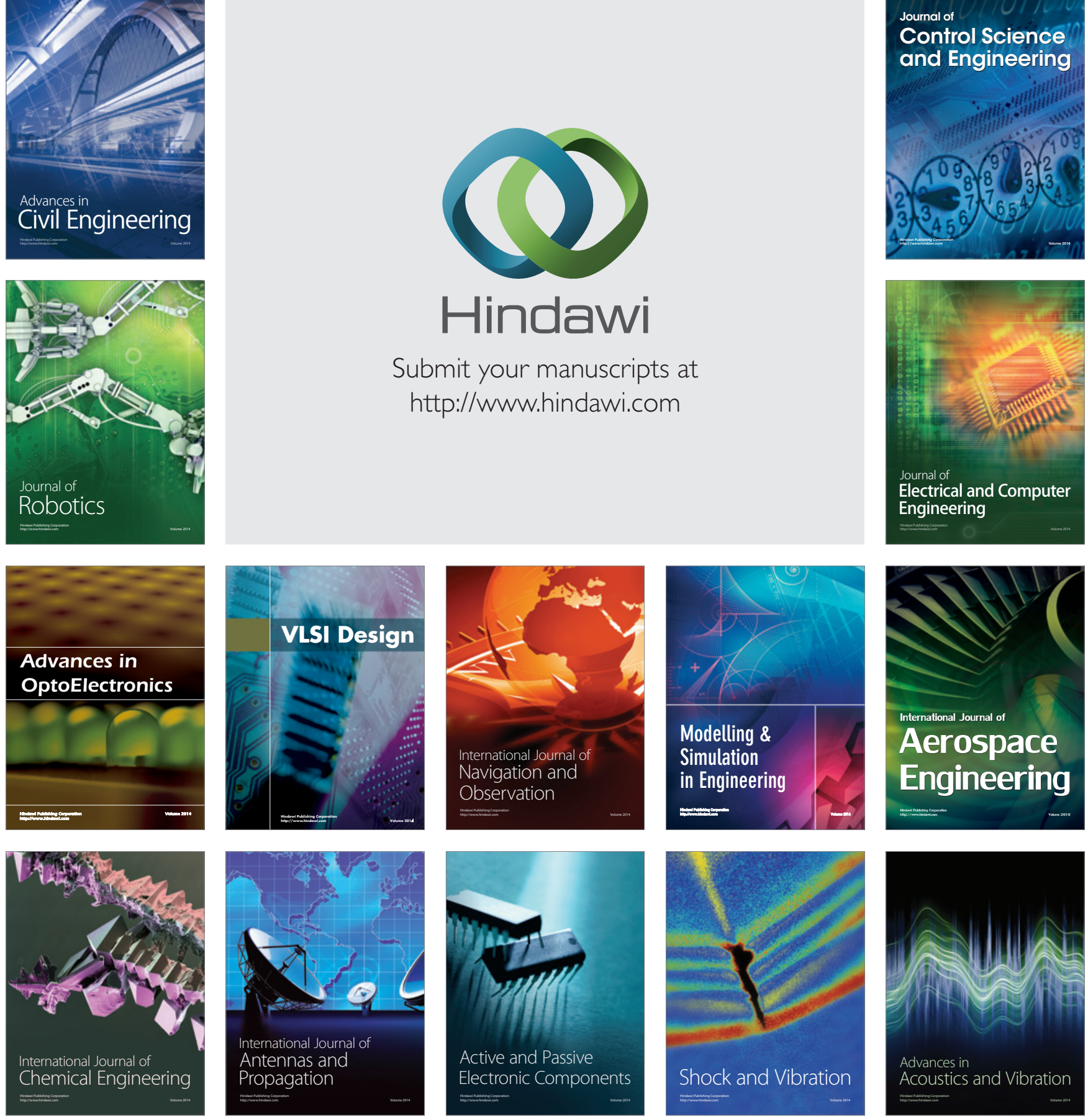